\title{
Os princípios cooperativistas e a identidade do movimento cooperati- vista em xeque
}

The cooperativist principles and the identity of the cooperativist movement in a bind

\begin{abstract}
Resumo
Este texto contribui na discussão sobre o atual dilema das cooperativas: manter a identidade do movimento ou buscar formas mais competitivas para se desenvolver. A abordagem proposta parte de um estudo "cross case" sobre a prática de princípios cooperativistas em quatro cooperativas de crédito do estado do Tocantins no Brasil de modo a subsidiar o debate. Ao final são apresentados alguns pontos para o avanço do debate.
\end{abstract}

Palavras-chave: Cooperativismo; Princípios Cooperativistas; Identidade Cooperativista.

\begin{abstract}
This paper contributes to the discussion on the current dilemma of cooperatives: maintain the identity of the movement or seek for more competitive forms to their development. The proposed approach is part of cross case study about the practice of cooperative principles in four credit unions in the state of Tocantins, in Brazil, in order to subsidize the debate. At the end, we present some points to advance the debate.
\end{abstract}

Keywords: Cooperativism. Cooperativist principles. Cooperativist identity.

Airton Cardoso Cançado ${ }^{1}$, Maria de Fátima Arruda Souza ${ }^{2} \&$ José Roberto Pereira ${ }^{3}$

${ }^{1}$ Universidade Federal do Tocantins, Doutor em Administração - airtoncardoso@yahoo.com.br

${ }^{2}$ Universidade Federal do Tocantins, Doutoranda em Administração - mfarrudasouza@gmail.com

${ }^{3}$ Universidade Federal de Lavras, Doutor em Sociologia - jrobpereira25@yahoo.com.br 


\section{Introdução}

$\mathrm{U}$ m cenário de constantes transformações no mundo dos negócios vem ocorrendo, estando em curso uma revolução sobre como você, seus colegas e concorrentes veem os clientes e, mais importante ainda, como os clientes estão vendo vocês e a eles mesmos. Até alguns anos atrás, o cliente era aquele indivíduo que comprava bens ou serviços de sua empresa. Parecia algo muito simples, mas a globalização começou a se movimentar rapidamente para todos os lados na década de 1990, com a entrada da internet no mercado principal (GREENBERG, 2001).

O primeiro International Summit of Cooperatives foi realizado em Quebéc no Canadá em outubro de 2012. No evento uma questão ficou bastante clara: as cooperativas devem se manter fiéis aos princípios cooperativistas ou buscar formas mais "competitivas" de ampliar seus negócios?

Algumas discussões durante o evento, inclusive no meio acadêmico, apontavam para uma dicotomia irreconciliável, enquanto outros grupos buscavam o equilíbrio entre as propostas. Obviamente a discussão não foi finalizada, apesar ou principalmente de alguns pontos de vista bastante apaixonados de ambos os lados.

O objetivo deste texto é discutir as possibilidades de conciliação entre essas duas propostas a partir da análise da prática dos princípios cooperativistas em cooperativas do estado do Tocantins, Brasil. Esta análise é construída por meio da discussão de quatro pesquisas sobre a prática dos princípios cooperativistas em cooperativas de crédito realizadas a partir de 2010 e conduzidas por pesquisadores da Universidade Federal do Tocantins UFT que fazem parte da Rede Brasileira de Pesquisadores sobre Cooperativismo - RBPC.

A seguir, apresenta-se um panorama das origens e evolução dos princípios cooperativistas, bem como uma contextualização do cooperativismo no Brasil e no estado do Tocantins, com destaque para o cooperativismo de crédito. As pesquisas já realizadas são analisadas como estudos de caso múltiplos (cross case), dada à proximidade de suas metodologias. A partir dos resultados dessas pesquisas realiza-se a discussão sobre uma possível aproximação dos pontos de vista descritos anteriormente. Ao final apresentam-se sugestões para novos trabalhos.

\section{Princípios Cooperativistas}

Os princípios cooperativistas foram idealizados quando da criação da Aliança Cooperativa Internacional em 1895. Nesse primeiro momento buscou-se na Rochdale Equitable Pioneers Society a inspiração para a criação desses princípios, que passam a ser a identidade da ACI, e por consequência, do movimento cooperativista em todo o mundo (SCHNEIDER, 1999; CANÇADO et al., 2012a).

A Cooperativa de Rochdale foi escolhida por ser reconhecida como a primeira cooperativa moderna. Antes dela existiram diversas experiências protocooperativas, algumas com algum sucesso. O que diferenciou Rochdale das outras experiências foi seu planejamento de quase um ano e a experiência de seus fundadores que já haviam participado de outras organizações e movimentos políticos que buscavam a melhoria da qualidade de vida. Porém, o grande diferencial foi que esse planejamento tomou forma de documento, hoje conhecido como estatuto. Com o êxito de Rochdale, muitas outras organizações nasceram inspiradas em seu estatuto, o que, de certa forma criou um "padrão informal" para a criação de novas organizações nesse formato (SCHNEIDER, 1999).

No $10^{\circ}$ Congresso da ACI, realizado na Basiléia em 1921, foi aprovado que as cooperativas seriam recomendadas a se orientar pelos princípios de Rochdale, tornando-se um critério para afiliação à ACI (SCHNEIDER, 1999). Assim, a ACI, munida de subsídios históricos e inspirada na experiência cooperativa em vários países, passou a assumir formal e explicitamente o legado de Rochdale. (SCHNEIDER, 1999, p.56)

Nos anos de 1937 (Paris), 1966 (Viena) e 1995 (Manchester), ocorreram reuniões da ACI que realizaram mudanças nos princípios cooperativistas (SCHNEIDER, 1999; PEREIRA et al., 2002), conforme Quadro 1.

Essa "evolução" dos princípios cooperativistas foi acompanhada por diversas consultas e discussões entre teóricos do cooperativismo, dirigentes de cooperativas e representantes das organizações cooperativas de todo o mundo, havendo sempre muito debate antes de alguma mudança (SCHNEIDER, 1999).

O congresso da ACI de 1995, realizado em Manchester, traz importantes mudanças nos princípios cooperativistas. A inclusão dos princípios "Autonomia e Independência" e "Preocupação com a Comunidade" indica que o cooperativismo está se atualizando. Com esta orientação, as cooperativas são orientadas a serem agentes autônomos, independentes e co-responsáveis pela comunidade.

Segundo Cançado et al. (2012a) as mudanças ocorridas nos princípios cooperativistas podem ser interpretadas como um reforço às suas características e não um desvio delas. Cabe ressaltar que muitos dos princípios, como a democracia, por exemplo, estão presentes desde o início.

A ICA (2014) define da seguinte forma os atuais princípios cooperativistas:

\section{Voluntary and Open Membership}

Co-operatives are voluntary organisations, open to all persons able to use their services and willing to accept the 


\begin{tabular}{|c|c|c|c|}
\hline \multicolumn{4}{|c|}{ PRINCÍPIOS COOPERATIVISTAS } \\
\hline \multirow{2}{*}{$\begin{array}{l}\text { Estatuto de } 1844 \\
\text { (Rochdale) }\end{array}$} & \multicolumn{3}{|c|}{ Congressos da Aliança Cooperativa Internacional } \\
\hline & 1937 (Paris) & 1966 (Viena) & 1995 (Manchester) \\
\hline $\begin{array}{l}\text { 1. Adesão Livre } \\
\text { 2. Gestão Democrática } \\
\text { 3. Retorno Pro Rata das } \\
\text { Operações } \\
\text { 4. Juro Limitado ao } \\
\text { Capital Investido } \\
\text { 5. Vendas a Dinheiro } \\
\text { 6. Educação dos } \\
\text { Membros } \\
\text { 7. Cooperativização } \\
\text { Global }\end{array}$ & $\begin{array}{l}\text { a) Princípios Essenciais } \\
\text { de Fidelidade aos } \\
\text { Pioneiros } \\
\text { 1. Adesão Aberta } \\
\text { 2. Controle ou Gestão } \\
\text { Democrática } \\
\text { 3. Retorno Pro-rata das } \\
\text { Operações } \\
\text { 4. Juros Limitados ao } \\
\text { Capital } \\
\text { b) Métodos Essenciais } \\
\text { de Ação e Organização } \\
\text { 5. Compras e Vendas à } \\
\text { Vista } \\
\text { 6. Promoção da } \\
\text { Educação } \\
\text { 7. Neutralidade Política } \\
\text { e Religiosa. }\end{array}$ & $\begin{array}{l}\text { 1. Adesão Livre } \\
\text { (inclusive neutralidade } \\
\text { política, religiosa, racial } \\
\text { e social) } \\
\text { 2. Gestão Democrática } \\
\text { 3. Distribuição das } \\
\text { Sobras: } \\
\text { a) ao desenvolvimento } \\
\text { da cooperativa; } \\
\text { b) aos serviços comuns; } \\
\text { c) aos associados pro- } \\
\text { rata das operações } \\
\text { 4. Taxa Limitada de } \\
\text { Juros ao Capital Social } \\
\text { 5. Constituição de um } \\
\text { fundo para a educação } \\
\text { dos associados e do } \\
\text { público em geral } \\
\text { 6. Ativa cooperação } \\
\text { entre as cooperativas } \\
\text { em âmbito local, } \\
\text { nacional e } \\
\text { internacional }\end{array}$ & $\begin{array}{l}\text { 1. Adesão Voluntária e } \\
\text { Livre } \\
\text { 2. Gestão Democrática } \\
\text { 3. Participação } \\
\text { Econômica dos Sócios } \\
\text { 4. Autonomia e } \\
\text { Independência } \\
\text { 5. Educação, Formação } \\
\text { e Informação } \\
\text { 6. Intercooperação } \\
\text { 7. Preocupação com a } \\
\text { Comunidade }\end{array}$ \\
\hline
\end{tabular}

Quadro 1 - Evolução dos Princípios Cooperativistas segundo a Aliança Cooperativa Internacional Fonte: Adaptado de Pereira et al. (2002).

responsibilities of membership, without gender, social, racial, political or religious discrimination.

\section{Democratic Member Control}

Co-operatives are democratic organisations controlled by their members, who actively participate in setting their policies and making decisions. Men and women serving as elected representatives are accountable to the membership. In primary co-operatives members have equal voting rights (one member, one vote) and co-operatives at other levels are also organised in a democratic manner.

\section{Member Economic Participation}

Members contribute equitably to, and democratically control, the capital of their co-operative. At least part of that capital is usually the common property of the co-operative. Members usually receive limited compensation, if any, on capital subscribed as a condition of membership.
Members allocate surpluses for any or all of the following purposes: developing their co-operative, possibly by setting up reserves, part of which at least would be indivisible; benefiting members in proportion to their transactions with the co-operative; and supporting other activities approved by the membership.

\section{Autonomy and Independence}

Co-operatives are autonomous, self-help organisations controlled by their members. If they enter into agreements with other organisations, including governments, or raise capital from external sources, they do so on terms that ensure democratic control by their members and maintain their co-operative autonomy.

\section{Education, Training and Information}

Co-operatives provide education and training for their members, elected representatives, managers, and employees so 
they can contribute effectively to the development of their co-operatives. They inform the general public - particularly young people and opinion leaders - about the nature and benefits of co-operation.

\section{Co-operation among Co-operatives}

Co-operatives serve their members most effectively and strengthen the co-operative movement by working together through local, national, regional and international structures.

\section{Concern for Community}

Co-operatives work for the sustainable development of their communities through policies approved by their members.

Esses princípios representam a identidade cooperativa por todo o mundo. No Brasil, a Organização das Cooperativas Brasileiras - OCB é filiada à ACI e também adota esses princípios. A Lei 5764/71, que regula o movimento cooperativista no Brasil, atende aos princípios cooperativistas, porém, como é anterior a 1995, os dois novos princípios não são considerados. A Constituição Brasileira de 1988 atualizou alguns aspectos dessa Lei, que passa a atender o princípio da Autonomia e Independência, mesmo antes dele figurar no rol dos princípios (CANÇADO et al., 2012a).

\section{Cooperativismo de crédito}

O cooperativismo de crédito tem sua origem na Alemanha, onde se constituíram segundo dois modelos: Schulze-Delitzsch e Raiffeisen. Nesses casos, as cooperativas não nasceram da organização popular, como na Inglaterra, mas do trabalho de dois homens oriundos da administração pública: Hermann Schulze, prefeito de Delitzsch (conhecido como Schulze-Delitzsch) e Friederich W. Raiffeisen, burgomestre de várias aldeias em torno de Neuwied, na Renânia (MEINEN; DOMINGUES; DOMINGUES, 2004).

Raiffeinsen, assessorado pelo pastor Müller, criou em 1848 a associação de Amparo aos Agricultores sem Recurso, também conhecida como Caixa Rural. A associação foi criada para que os agricultores tivessem outra opção além do agiota local. A ideia era reunir as economias dos produtores com mais recursos e emprestar aos agricultores menos favorecidos sem a perspectiva do ganho abusivo. A organização era gerida democraticamente e reunia pessoas que se conheciam em uma área de atuação restrita. Os resultados não eram distribuídos. Embora não fosse efetivamente uma cooperativa, este seria o modelo para a futura atividade cooperativista de Raiffeinsen (MEINEN, DOMINGUES; DOMINGUES, 2004; PINHEIRO, 2005).
Em 1850, Herman Schulze criou as Caixas de Socorro na cidade de Delitzsch com uma perspectiva próxima à da criação da Caixa Rural, mas em um contexto urbano, conhecidas como Schulze-Delitzsch. As diferenças em relação ao modelo anterior são: previsão de retorno das sobras líquidas, área de atuação não restrita e a remuneração dos dirigentes (MEINEM; DOMINGUES; DOMINGUES, 2004; PINEIRO, 2005).

Em 1865, com a disseminação dos modelos alemães de cooperativismo de crédito pelo mundo, Luigi Luzzatti em Milão, na Itália, realiza adaptações dos modelos anteriores e funda o Banco Popular, o primeiro Banco Cooperativo (MEINEM; DOMINGUES; DOMINGUES, 2004).

Inspirado nos modelos anteriores, Alphonse Desjardins idealizou um novo modelo de cooperativa de crédito. A primeira cooperativa constituída por Desjardins foi em Quebéc no Canadá em 1900. A principal diferença do modelo de Desjardins é que nesta cooperativa há alguma espécie de vínculo entre os cooperados: funcionários de uma mesma empresa, profissionais da mesma classe, membros de um clube, etc (PINHEIRO, 2005).

Em seguida, será apresentado o contexto do cooperativismo no Brasil e no Tocantins, com especial atenção para as cooperativas de crédito.

\section{O Cooperativismo no Brasil e no Tocan- tins}

O cooperativismo no Brasil tem início em 1889 na cidade de Ouro Preto, então capital do estado de Minas Gerais. Posteriormente o cooperativismo chega a outros estados como Pernambuco, São Paulo e Rio Grande do Sul. Esse movimento contou com a intensa participação de imigrantes europeus, sobretudo alemães e italianos (OCB, 2014a).

Inicialmente, a grande extensão do país dificultou a interiorização do cooperativismo. Porém, o cooperativismo se consolidou nos locais onde se estabeleceu, apesar de enfrentar diversos problemas. Em 1969 é criada a Organização das Cooperativas Brasileiras e em 1971 é promulgada a Lei do Cooperativismo. Recentemente, em 2012, uma legislação específica para o cooperativismo de trabalho foi aprovada, dadas as divergências de entendimento legal relacionadas a essas cooperativas (OCB, 2104a).

Em 1998 foi criado o Serviço Nacional de Aprendizagem do Cooperativismo, o SESCOOP, porém o início de seu funcionamento foi em 2002. Os objetivos do SESCOOP são: ensino de formação profissional baseada nos valores e princípios do cooperativismo; organização e promoção social para os cooperados e comunidades do entorno das cooperativas; e monitoramento e orientação a cooperativas. A partir de então, o cooperativismo ganha novo fôlego no Brasil. (OCB, 2014b). 
Para o cooperativismo de crédito, a partir de Resolução do 3.106 de 2003 foi permitida novamente a constituição de cooperativas de livre admissão (modelo Luzzatti), com algumas restrições. Diversas outras resoluções posteriores foram editadas no sentido de diminuir essas restrições (PINHEIRO, 2005). Esse contexto facilitou as fusões e incorporações no ramo de crédito, bem como tornou possível a sobrevivência de outras cooperativas no competitivo mercado financeiro.

O número de cooperativas no Brasil passou por dois períodos de queda. O primeiro entre 2002 e 2004, no início do primeiro governo Lula, causado pelo medo de mudanças drásticas na economia e pelo risco de investir no Brasil. O ramo que mais recuou foi o agropecuário, onde mais de 220 cooperativas deixaram de existir. Porém, a quantidade de cooperados no ramo agropecuário se manteve constante e o número de funcionários dessas cooperativas aumentou cerca de 10\%. Esta diminuição, em grande parte, é resultado de fusões e incorporações dessas cooperativas, buscando economia de escala e de escopo. Pode-se argumentar que o princípio da intercooperação poderia contribuir nesse sentido, porém, a esses ganhos, somam-se a queda dos custos de transação e de agência derivados de uma gestão central.

A segunda etapa de declínio da quantidade de cooperativas no Brasil ocorreu entre 2008 e 2011. Apesar de serem constatadas algumas fusões e incorporações, elas não podem ser consideradas as principais responsáveis. O grande responsável pelo declínio do número total de cooperativas no país foi o ramo de trabalho. De 2001 a 2011 a quantidade de cooperativas de trabalho diminui em cerca de 50\%, passando de 1.849 para 966 . A principal causa para esse declínio foi relacionada à precariedade do marco legal dessas cooperativas e do entendimento do judiciário que tais cooperativas eram fraudulentas. Esse foi um dos motivos da criação, em 2012, de uma legislação específica para o ramo. Por outro lado, as cooperativas do ramo transporte foram de um total de 548 para 1.082 no mesmo período. Nesse caso, grande parte dessa evolução pode ser atribuída ao transporte alternativo urbano e interurbano e parte pela terceirização de frotas de grandes empresas.

No caso do Tocantins, como se pode notar, a quantidade de cooperativas é pequena. O estado foi criado pela constituição federal de 1988 a partir do desmembramento do estado de Goiás.

O comportamento do cooperativismo tocantinense guarda algumas semelhanças com os números nacionais do setor. O número de cooperativas no Tocantins também passou por dois períodos de decréscimo, equivalentes ao nacional.

No primeiro período de queda (2002-2003), as cooperativas agropecuárias foram de 40 para 13, acompanhadas pela queda do número de cooperados, de 3.365 para 1.208 e de funcionários, de 1.025 para 578 . Nesse caso, as cooperativas encerraram suas atividades. Outros ramos afetados nesse período foram as cooperativas de trabalho, que foram de 10 para 4 e as de mineração que foram de 4 para apenas 1 . Nesses casos também, as cooperativas encerraram as atividades.

No período entre 2009 e 2010, o responsável pela queda no número de cooperativas foi novamente o ramo agropecuário, porém, dessa vez, houve mais fusões e incorporações. Neste caso, o número de cooperados não caiu na mesma proporção (passando de 3.549 para 3.219) e a quantidade de funcionários das cooperativas agropecuárias aumentou de 314 para 445 .

\subsection{Cooperativismo de crédito no Brasil}

As cooperativas de Crédito no Brasil são reguladas pelo Banco Central do Brasil - BCB, pois fazem parte do Sistema Financeiro Nacional. Desta forma, além da legislação cooperativista, as cooperativas de crédito atendem às resoluções do BCB e estão sujeitas à sua fiscalização.

Durante o século $X X$, o cooperativismo de crédito no país passou por diversas fases. A primeira cooperativa foi constituída em 1902 no Rio Grande o Sul. Seus resultados foram tão relevantes que logo o cooperativismo de crédito se espalhou pelo estado (PAGNUSSATT, 2004).

O cooperativismo de crédito brasileiro foi inspirado em três modelos: no modelo alemão Raiffeisen, nas cooperativas rurais, no modelo de cooperativas de classe canadense Desjardins, nas cooperativas urbanas e no modelo italiano Luzzatti. Na década de 1950, foi proibida a constituição das cooperativas do tipo Luzzatti.

Um grave revés acontece por volta de 1965 (a tomada do poder pelos militares foi em 1964), quando é realizada uma reformulação da legislação bancária e os bancos oficiais foram escolhidos como veículo de concessão do crédito agrícola. Nesse contexto histórico, as cooperativas ainda sofreram uma restrição em seu espectro societário e operacional, o que levou ao fechamento de diversas cooperativas.

No início da década de 1980, também no Rio Grande do Sul, o cooperativismo se organizava de forma sistêmica por meio de uma central, a Cooperativa Central de Crédito Rural do Rio Grande do Sul Ltda. - COCECRER. A partir desta iniciativa, o cooperativismo de crédito, mesmo sob um marco regulatório desfavorável, renasce no país com a criação de novas cooperativas singulares e centrais e em 1986 é criada a Confederação Brasileira das Cooperativas de Crédito - CONFEBRÁS (MEINEN; DOMINGUES; DOMINGUES, 2002).

Em 1992, começam as grandes conquistas do cooperativismo de crédito. Neste ano as cooperativas de crédito foram autorizadas a captarem depósitos a prazo, aumentando o volume dos recursos administrados. Em 1994, é publicada a autorização para a criação de Postos de Atendimento Cooperativo (equivalentes a agências/ filiais) e, no ano seguinte, as cooperativas são autoriza-

RGC - Vol. 01, Nº2, $2^{\circ}$ Sem. 2014, Págs. 63-72 
das a constituir bancos cooperativos, sendo o primeiro constituído no mesmo ano (PAGNUSSATT, 2004).

Já no início do século XXI, o Conselho Monetário Nacional passa a reconhecer o papel das cooperativas centrais na capacitação e controle das afiliadas, além da prestação de contas ao $\mathrm{BCB}$. $\mathrm{O}$ avanço mais significativo ocorre em 2003, com a permissão da livre associação, que consolidou a organização sistêmica (centrais e bancos cooperativos) das cooperativas brasileiras. A partir daí, outras resoluções foram tomadas, facilitando cada vez mais a criação de cooperativas de livre admissão e a transformação das já existentes em cooperativas com esta configuração (PAGNUSSATT, 2004).

A tendência atual do cooperativismo de crédito brasileiro caminha no sentido de fusões e incorporações de cooperativas. Este movimento deve se tornar mais forte no decorrer desta década. Além disso, as cooperativas também têm caminhado no sentido da livre associação.

No caso das cooperativas de crédito no Brasil, em 2001 havia 1.038 e em 2011 elas somavam 1.042. Em relação à quantidade de cooperados em cooperativas de crédito, o número foi de cerca de um milhão em 2001 para mais de quatro milhões e meio em 2011. Para cada cooperado associado em 2001, cerca de quatro novos cooperados ingressaram nas cooperativas em 10 anos, pois o número de cooperativas se manteve constante. Outra informação importante é que esse crescimento foi contínuo no período. Em relação ao número de funcionários, as cooperativas de crédito contavam com cerca de 20.000 em 2001, chegando a mais de 56.000 em 2010, porém, em 2011 esse número cai para um número próximo de 34.000. Aqui, nota-se mais claramente o papel das fusões e incorporações, mas também deve ser contabilizado o papel da automação, potencializado por cooperativas mais robustas e organizadas. Entre 2010 e 2011 o número de cooperativas se manteve praticamente estável e o de cooperados cresceu em mais de 650.000 (OCBTO, 2014).

\subsection{O cooperativismo de crédito no Tocantins}

O cooperativismo de crédito do Tocantins passou por alguns momentos de crescimento e decrescimento, que denotam tanto o fechamento, a criação e a incorporação de cooperativas no estado. Porém, a tendência é de aumento do número de cooperados e de funcionários, seguindo o mesmo caminho dos números nacionais.

Atualmente são 3 cooperativas de crédito em atividade: Sicredi União Cerrado, Sicoob Credipar e Unicredi Centro Brasileira. Apesar do pequeno número, as cooperativas contam com 17 Postos de Atendimento Cooperativo.

Essas cooperativas têm mantido um constante crescimento, tanto do número de cooperados, quanto de funcionários. A tendência apresentada no estado é a da abertura de mais Postos de Atendimento Cooperativo e não da criação de novas cooperativas, conforme o próprio direcionamento do $\mathrm{BCB}$.

\section{A prática dos princípios cooperativistas nas cooperativas de crédito do Tocantins}

Foram analisados 4 estudos realizados entre $2010 \mathrm{e}$ 2012 em cooperativas de crédito do Tocantins, sendo 3 trabalhos de conclusão de curso de graduação em Administração: Jesus (2010), Silva (2011) e Abreu (2011); e uma dissertação de mestrado em Desenvolvimento Regional, Bertuol (2012). Os trabalhos de graduação tiveram como objetivo analisar a prática dos princípios cooperativistas na ótica dos dirigentes, enquanto a dissertação também considerou a opinião dos funcionários e cooperados. Todos os trabalhos foram realizados no âmbito da Universidade Federal do Tocantins - UFT.

A análise que será feita vai considerar os resultados finais relativos aos pontos fortes e fracos encontrados pelos pesquisadores em relação à prática dos princípios cooperativistas.

O trabalho de Jesus (2010) se refere à cooperativa Sicredi Araguaia Tocantins, que posteriormente foi incorporada e passou a fazer parte da cooperativa Sicredi União Cerrado. A pesquisa de Silva (2011) foi realizada na Sicoob Credipar e a de Abreu (2011) na cooperativa Sicoob Meio Norte. Em 1ํo de dezembro de 2011 a cooperativa Sicoob Credipar incorpora a cooperativa Sicoob Meio Norte. O trabalho de Bertuol (2012) se refere à cooperativa Sicoob Credipar depois dessa incorporação.

Quanto ao princípio da Adesão Livre e Voluntária, as cooperativas que antes das incorporações eram cooperativas do tipo Desjardins (de classe ou segmento profissional), com as incorporações passaram a ser de livre admissão (modelo Luzzatti), à exceção da Unicredi, que é vinculada a profissionais da saúde. Esta abertura favoreceu a ampliação do quadro de cooperados e do próprio movimento das cooperativas.

Quanto ao princípio da Gestão Democrática, as cooperativas têm um número muito baixo de participação dos cooperados em assembleias. Esse ponto é bastante relevante, pois, à medida que a cooperativa amplia o número de cooperados, aumenta suas possibilidades de sobrevivência e crescimento. Por outro lado, com uma maior quantidade de cooperados fica mais difícil operacionalizar a Gestão Democrática, que pode caminhar para uma democracia representativa, com todos os problemas oriundos dessa opção, como a dificuldade de representar interesses divergentes e o próprio desconhecimento em relação aos interesses dos representados (LÜCHMANN, 2007). Uma das alternativas encontradas pelas cooperativas de crédito do Tocantins é a realização de pré-assembleias, no intuito de informar e dar voz aos cooperados, possibilitando a participação direta, em conjunto com a representação. Porém, essas pré-as- 


\begin{tabular}{|l|l|l|}
\hline Cooperativa & Sede & Postos de Atendimento Cooperativo \\
\hline $\begin{array}{l}\text { Sicredi União } \\
\text { Cerrado }\end{array}$ & Dianópolis & $\begin{array}{l}\text { Palmas, Araguatins, Pedro Afonso e Luis Eduardo } \\
\text { Magalhães (estado da Bahia) }\end{array}$ \\
\hline Sicoob Credipar & $\begin{array}{l}\text { Paraíso do } \\
\text { Tocantins }\end{array}$ & $\begin{array}{l}\text { Barrolândia, Pium, Marianopolis, Miranorte, Divinópolis, } \\
\text { Dois Irmãos, Cristalândia, Gurupi e Palmas }\end{array}$ \\
\hline $\begin{array}{l}\text { Unicredi Centro } \\
\text { Brasileira }\end{array}$ & Palmas & Araguaína \\
\hline
\end{tabular}

Quadro 2 - Cooperativas de crédito e Postos de Atendimento Cooperativo no Tocantins. Fonte: OCBTO (2014).

sembleias ainda estão em fase de implantação ou foram realizadas poucas vezes.

Em relação ao princípio da Participação Econômica dos Membros, as cooperativas não apresentam problemas significativos; as regras são seguidas sob a vigilância do BCB e a supervisão das cooperativas centrais de crédito, ao qual as cooperativas são ligadas. Com a possibilidade de oferecerem diversos produtos como cartão de crédito, seguros, títulos de capitalização, dentre outros, as cooperativas de crédito estão aptas a melhorar a prestação de serviços aos seus cooperados. Outra constatação importante é a proliferação dos pontos de atendimento que aumenta a capilaridade das cooperativas e favorece a ampliação do quadro de associados e as possibilidades de operações financeiras.

O princípio da Autonomia e Independência traz uma tensão ao debate. Por um lado, as cooperativas devem seguir a legislação e as resoluções do $\mathrm{BCB}$ por fazerem parte do Sistema Financeiro Nacional. A fiscalização é praticamente online e realizada por intermediação da cooperativa central. Se por um lado a cooperativa central dá segurança para as cooperativas, por outro, ela direciona muitas das decisões da cooperativa, limitando sua autonomia. Ainda assim, a recomendação do BCB é de incentivar a filiação das cooperativas a centrais, no intuito de fortalecer o sistema como um todo e diluir riscos.

O quinto princípio cooperativista Educação, Formação e Informação pode ser considerado como essencial para o bom desempenho das cooperativas. A grande maioria dos entrantes em organizações cooperativas no Brasil desconhece as diferenças desse tipo de empreendimento em relação às empresas comerciais com fins lucrativos. Nesse sentido, a observação dos princípios e a fidelidade dos cooperados à cooperativa dependem do trabalho contínuo de educação cooperativista. As cooperativas tocantinenses têm à sua disposição recursos próprios e do SESCOOP para a realização desses cursos, porém, o nível de participação dos cooperados nessas atividades é considerado baixo segundo os estudos analisados. As pesquisas analisadas indicam também que a comunicação entre cooperativa e cooperado é deficiente. Apesar da existência de canais de comunicação entre a cooperativa e seus cooperados, estes não estão sendo efetivos. Em outras palavras, a estratégia de comunicação entre cooperativa e cooperado precisa ser revista.

O princípio da Intercooperação ainda é pouco praticado pelas cooperativas do Tocantins. Muitas vezes se restringe a eventos e formações promovidos pelas cooperativas centrais e SESCOOP. Não há parcerias com perspectiva de longo prazo. Essa situação justifica as fusões e incorporações que ocorreram recentemente no estado, pois como não há alianças, o caminho para economia de escopo e de escala passa a ser a junção de cooperativas.

Por fim, o princípio da Preocupação com a Comunidade também apresenta alguns entraves à sua prática. A cooperativa que melhor se organiza nesse sentido é a Sicoob Credipar, com alguns programas estruturados voltados para os cooperados (SILVA, 2011; BERTUOL, 2012). Porém, as ações em relação à comunidade podem ser consideradas como pontuais e assistencialistas. Essas ações são importantes, mas não têm um planejamento de longo prazo e são apenas paliativas, com baixo impacto no desenvolvimento das comunidades. Muitas cooperativas ainda confundem o princípio da Preocupação com a Comunidade com responsabilidade social para cooperativas, conforme já discutido por Cançado et al. (2012b) no International Summit of Cooperatives de 2012. Esse entendimento pode dar a impressão que a cooperativa deve "ajudar quando pode" e de "maneira pontual" a comunidade que a cerca, quando na verdade essa relação com a comunidade é um dos pilares do cooperativismo.

De forma geral, as cooperativas de crédito do estado do Tocantins vêm buscando praticar os princípios cooperativistas. Ainda há um longo caminho, mas parece que as cooperativas estão no caminho certo. Os problemas identificados estão muito relacionados à ampliação da cultura cooperativista junto aos cooperados, pois as distorções advindas desse desconhecimento, de maneira

RGC - Vol. 01, $\mathrm{N}^{\circ} 02,2^{\circ}$ Sem. 2014, Págs. 63-72 
geral, são as causas para os problemas apontados.

\section{Por uma possibilidade de desenvolvi- mento do cooperativismo sem perda da identidade}

Com relação a uma possível dicotomia entre a prática dos princípios cooperativistas e a aproximação com o mercado no intuito de ampliar o potencial de competição das cooperativas, pode-se afirmar que temos dois aspectos interdependentes. É urgente se ampliar a cultura da cooperação entre os cooperados dessas cooperativas, porém isso demanda a aplicação do princípio da Educação, Formação e Informação que sofre com o problema da comunicação. Assim, a comunicação tal como realizada hoje (os canais existem e são usados) não está surtindo o efeito desejado. $\mathrm{O}$ apelo à participação e à tomada de maiores responsabilidades em relação à cooperativa soa estranho para quem não conhece o funcionamento dessas organizações. Um bom começo seria rever a comunicação no sentido de sensibilizar esse público da mensagem a ser apresentada. Não é uma tarefa fácil, mas ela deve ser realizada, sob pena de as cooperativas se aproximarem do mercado pelo próprio desconhecimento de outras possibilidades relacionadas à natureza da organização cooperativa.

Outro dilema a ser enfrentado, que está fortemente ligado ao anterior, é o tamanho da organização cooperativa. De forma geral, quanto maior a cooperativa, maiores as possibilidades de sobrevivência e desenvolvimento (economia de escala e escopo), principalmente no caso das cooperativas de crédito. Por outro lado, quanto mais cooperados, mais difícil se torna a participação direta. Quando as cooperativas conseguirem melhorar sua comunicação e fortalecer a cultura da cooperação, os cooperados estarão mais dispostos a participar e então o tamanho da organização pode ser um empecilho à concretização dessa participação. Paralelamente às ações de melhoria da comunicação devem ser ampliados também os canais de participação como as pré-assembleias, que já existem, ou mesmo utilizando as tecnologias da informação e comunicação.

\section{Sugestões de novos trabalhos}

Esse texto buscou contribuir para o debate acerca do dilema atual vivido pelas cooperativas, entre reforçar ou flexibilizar sua identidade enquanto organizações baseadas em princípios específicos. Foram apontados alguns problemas relacionados à prática dos princípios cooperativistas em cooperativas de crédito do estado do Tocantins, Brasil de forma a apoiar a discussão.

Apesar dos problemas das demais cooperativas brasileiras de todos os ramos serem, de certa forma, próximos a esses, são necessários outros estudos para confirmar essa assertiva e avançar na discussão. Sugerem-se novos estudos em cooperativas brasileiras e de outros países para ampliar esse debate. Devem ser incluídas nessas novas pesquisas as cooperativas que participaram de processos de fusão e incorporação, bem como as que encerraram suas atividades para entender os motivos que levaram a estas situações.

\section{Referências}

ABREU, P. C. Prática dos princípios cooperativistas: um estudo de caso em Palmas-TO. TCC (Graduação em Administração). Palmas: UFT, 2011.

BERTUOL, R. A prática dos princípios cooperativistas: um estudo de caso no Tocantins. Dissertação (Mestrado em Desenvolvimento Regional). Palmas: UFT, 2012.

CANÇADO, A. C.; A. S. RIGO ; J. R. PEREIRA; M. C. H. GONTIJO. (2012). Movimento e Princípios Cooperativistas: Evolução e Reflexões para Novos Estudos. In CANÇADO, A. C.; F. G. TENORIO ; J. T. SILVA JR (orgs.). Gestão Social: aspectos teóricos e aplicações. Ijuí: UNIJUí, 2012a.

CANÇADO, A. C.; RIGO, A. S.; SILVA JR, J. T.; SOUZA, M. F. A. Beyond Mimicry: a critique of "Social Responsibility" in Co-operatives. In BRASSARD, M.J.; Molina, E. (orgs.) (2012). The Amazing Power of Cooperatives. Texts selected from the international call for paper proposals, Québec, International Summit of Cooperatives, 2012b.

ICA - INTERNATIONAL CO-OPERATIVE ALIANCE. Co-operative identity, values \& principles. Disponível em < http://ica.coop/en/what-co-op/co-operative-identity-values-principles > Acesso 24 jan. 2014.

JESUS, W. L. Princípios cooperativistas: analisando sua aplicação na cooperativa de crédito Sicredi - Araguaia Tocantins em Palmas - TO. TCC (Graduação em Administração). Palmas: UFT: 2011.

LÜCHMANN, L. H. H. A representação no interior das experiências de participação. Lua Nova, n.70, p. 139-170, 2007.

MEINEN, Ê.; DOMINGUES, J. N.; DOMINGUES, J. A. S. Cooperativas de Crédito no Direito Brasileiro. Porto Alegre: Sagra Luzzatto, 2002.

OCB - Organização e SINDICATO DAS COOPERATIVAS BRASILEIRAS. Movimento livre da influência do Estado. Disponível em < http://www.ocb.org.br/ 
site/cooperativismo/evolucao_no_brasil.asp > Acesso 24 jan. 2014a.

OCB - Organização e SINDICATO DAS COOPERATIVAS BRASILEIRAS. Áreas de atuação prioritárias. Disponível em < http://www.ocb.org.br/site/sescoop/ atuacao.asp > Acesso 24 jan. 2014b.

PAGNUSSATT, A. Guia do cooperativismo de crédito - organização governança e políticas corporativas. Porto Alegre: Sagra Luzzatto, 2004.

PEREIRA, J. R. et al. Organização da sociedade através das cooperativas de trabalho: abordagem dos problemas e perspectivas. Relatório final de pesquisa - FAPEMIG. Viçosa: UFV, 2002.

PINHEIRO, M. A. H. Cooperativas de Crédito - História da evolução normativa no Brasil. 3. Ed. Brasília, 2005.

SCHNEIDER, J. O. Democracia, participação e autonomia cooperativa. 2. ed. São Leopoldo: UNISINOS, 1999. $496 \mathrm{p}$.

SILVA, F. R. Prática dos princípios cooperativistas: um estudo de caso em uma cooperativa de crédito do Tocantins. TCC (Graduação em Administração). Palmas: UFT, 2011. 
